\title{
Estimation of Vehicle Sideslip Angle Based on Feedback Observer
}

\author{
Shaosong Li, Luping Guo, Shunhang Zheng, Guangjun Li, Xu Yan, Yusheng Yang and Zhixin Yu* \\ School of Mechanical and Electrical Engineering Changchun University of Technology, China \\ ${ }^{*}$ Corresponding author
}

\begin{abstract}
Vehicle sideslip angle is a significant parameter for vehicle stability control. Based on the 2-DOF vehicle dynamic model, a feedback observer is built with the correction item. The estimation accuracy of the sideslip angle is improved by adjusting the feedback gain of the feedback term in the feedback observer. At the same time, the computational burden is reduced by using the three-map of tire lateral force based on the magic formula (MF) model. The simulation results show that the feedback gain plays an important role in improving accuracy of sideslip angle estimation. The estimation method can be applied to the vehicle stability control system.
\end{abstract}

Keywords-sideslip angle estimation; three-map of tire lateral force; feedback observer; observer gain

\section{INTRODUCTION}

The vehicle stability control enables the vehicle to have good handling stability and active safety, which is an efficient way to reduce vehicle accidents $[1,2]$. In the stability control algorithm, sideslip angle and yaw rate are important indexes to represent vehicle stability and are the main control variables [3]. However, unlike yaw rate, sideslip angle cannot be measured directly in production vehicles because of accuracy and cost issues.

In the literature, there have been many efforts to estimate sideslip angle. Reference $[4,5]$ use simple linear tire model to provide the required tire force to estimate sideslip angle. Unfortunately, because of the particular shape of the tire lateral force characteristics, the tire slip angles, and consequently also the vehicle slip angle, may increase quite rapidly without corresponding increase in lateral forces in nonlinear range. Therefore, the estimation accuracy of sideslip angle will be inevitably affected by using a linear tire model. Bevly et al. used GPS velocity information in conjunction with inertial measurement units (IMUs) to obtain sideslip angle [6]. Unlike indirect measurement of sideslip angle using lateral acceleration or yaw rate, direct measurement of sideslip angle via optical or GPS sensors are associated with problems of cost, and reliability. In addition, Zhang developed a side slip angle observer by adopting filter method. Huang developed a neural network algorithm and Shi proposed a Fuzzy logic method, which can estimate sideslip angle more accurately[7, 8]. Milanese et al. set up a nonlinear estimator and tested it for several steering maneuvers [9]. These estimation methods are based on the nonlinear estimation model. The model has many dimensions and the nonlinear estimation algorithm is complex, which would be limited in the application of the actual control system.

In this paper, in order to improve real-time, a feedback observer is proposed to estimate the sideslip angle based on a 2 -DOF vehicle model. In the design procedure, we consider the nonlinear behavior of tire force between tire and road and obtain tire lateral force by using a three-map to reduce the computational burden. Finally, simulation experiments demonstrate the effectiveness of the proposed feedback observer.

\section{VEHICLE DYNAMICS MODEL}

\section{A. Vehicle Modele}

To estimate sideslip angle, the vehicle model used is a 2DOF vehicle model which can accurately reflect the lateral characteristics with smaller computing load. The 2-DOF vehicle model is illustrated in Fig. 1. Assuming pure lateral slip and constant longitudinal speed, the lateral and yaw dynamics can be expressed as:

$$
\begin{aligned}
& F_{y f}+F_{y r}=m(\dot{v}+u \cdot r) \\
& a F_{y f}-b F_{y r}=I_{z} \cdot \dot{r}
\end{aligned},
$$

where $m$ is the mass of the vehicle; $a$ and $b$ are the distances from the front axle and rear axle to the vehicle gravity center, respectively, $I_{z}$ is the vehicle inertia about the vertical axis, $u$ is the longitudinal velocity, $v$ is the lateral velocity, $F_{y f}$ and $F_{y r}$ are the lateral tire forces, $r$ is the yaw rate of the vehicle and $\delta$ is the front wheel steering angle. By using simple kinematics, we can write $\alpha_{f}$ and $\alpha_{r}$, the slip angle $\beta$ of front and rear tires, in terms of $u, v$, and $r$ :

$$
a_{f}=b+\frac{a r}{u}-d, a_{r}=b-\frac{b r}{u} .
$$

where $\alpha$ and $\alpha \mathrm{r}$ are sideslip angle of front wheel and rear wheel.

\section{B. Tire Model}

The magic formula (MF) model is adopted in this study [10], which is expressed as: 


$$
Y(x)=D \sin (C \arctan (B x-E(B x-\arctan (B x))))+S_{V},
$$

with $x=X+S_{h}$,

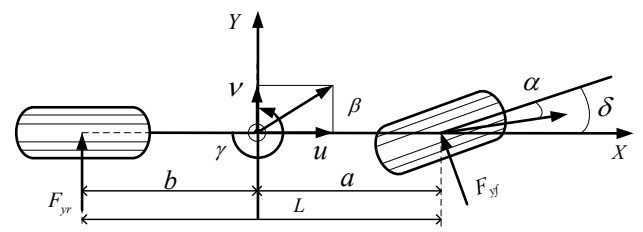

FIGURE I. 2-DOF VEHICLE DYNAMICS MODEL

where $\mathrm{Y}(\mathrm{x})$ is the lateral force $F_{y}$; and $X$ is the slip angle, $C, D$, $B$ and $E$ are derived from experiments.

$$
\left\{\begin{array}{l}
C=A_{0} ; \\
D=A_{1} \cdot F_{z}^{2}+A_{2} \cdot F_{z} ; \\
B=\frac{A_{3} \cdot \sin \left(\arctan \left(F_{z} / A_{4}\right) \cdot 2\right)}{C D} \\
E=A_{6} \cdot F_{z}+A_{7} ; \\
S_{n}=A_{9} \cdot F_{z}+A_{10} ; \\
S_{V}=A_{12} \cdot F_{z}+A_{3} ;
\end{array}\right.
$$

where $F_{z}$ is the vertical load of tire. The parameters of $A_{0}-A_{13}$ obtained by fitting the experimental data. And $F_{y}$ is a function of $F_{z}$, as shown in Fig. 2 .

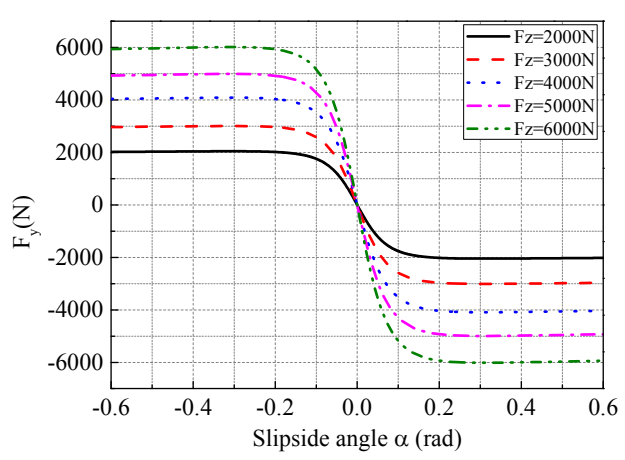

FIGURE II. LATERAL FORCE

The tire lateral force map is established as a three-dimensional look-up table which is a function of the friction coefficient and slip angle, as shown in Fig. 3.

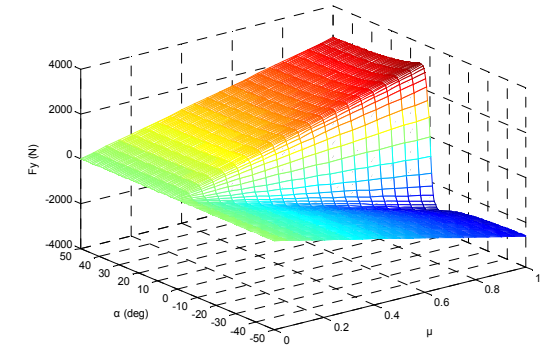

FIGURE III. THREE-MAP OF TIRE LATERAL FORCE

\section{ESTIMATION OF VEHICLE SIDESLIP ANGLE}

In order to analyze the estimation of sideslip angle and considering the existing on-board sensors, this paper makes the following assumptions: lateral acceleration of vehicle $a_{y}$, yaw rate $r$ and friction coefficient $\mu$ are known. The estimation scheme based on feedback observer proposed is shown as Fig. 4.

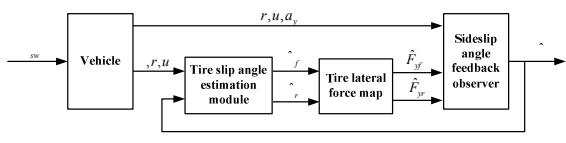

\section{FIGURE IV. ESTIMATION SCHEME BASED ON FEEDBACK OBSERVER}

Prior to beginning estimation, the steering wheel angle $\delta_{s w}$ is excitation input and the initial $\beta_{0}$ sideslip angle are set to zero (i.e. the vehicle is driving straight). Front and rear tire slip angle can be obtained through the tire slip angle. Front and rear lateral force can be obtained through the three-map of tire lateral force according to front and rear tire slip angle. And with the measured vehicle state information, the feedback observer can estimate sideslip angle.

\section{A. Feedback Observer Design}

From the geometric relation of Fig. 1, the sideslip angle is obtained as follows:

$$
b=\frac{v}{u}
$$

To update estimate of slip angle, we can derive the feedforward term of the update equation by taking the (1) and (4):

$$
b^{\&}=\frac{1}{m \times u}\left(F_{y f}+F_{y r}\right)-r
$$

The lateral acceleration of the vehicle can be directly measured by the sensor. Therefore, the lateral acceleration is used as an observed output of the system: 


$$
y=a_{y}
$$

On the basis of (2), (3) and (5), the dynamic equations of vehicle body are expressed as state space equation as follows:

$$
b^{\&} f(b), y=g(b)
$$

Since the sideslip angle cannot be measured directly, a feedback observer is designed:

$$
b^{\&}=f(\hat{b})+K(y-\hat{y}),
$$

where $K$ is an observer gain.

At the same time, the lateral force of the vehicle is calculated by lateral acceleration. Taking the difference between the measured lateral acceleration and the estimated value of vehicle lateral force as the correction term, the feedback observer of sideslip angle $\beta$ is designed as follows:

$$
\dot{\hat{\beta}}=\frac{1}{m \cdot u}\left(\hat{F}_{y f}+\hat{F}_{y r}\right)-r+K\left(m \cdot a_{y}-\hat{F}_{y f}-\hat{F}_{y r}\right)
$$

where $\hat{F}_{y f}, \hat{F}_{y r}$ can be obtained through the three-map of tire lateral force according to front and rear tire slip angle. $\hat{a}_{f}, \hat{a}_{r}$, $\hat{a}_{f}, \hat{a}_{r}$ are calculated as follows:

$$
\hat{a}_{f}=\hat{b}+\frac{a r}{u}-d, \hat{a}_{r}=\hat{b}-\frac{b r}{u}
$$

\section{SIMULATION AND DISCUSSION OF RESULTS}

In order to verify the proposed estimation methods Simulations are conducted on the basis of Carsim and Matlab/Simulink. CarSim is a special software for the simulation of vehicle dynamics, which is widely used in modern vehicle control system.

In order to render the responses of CarSim model to be similar to that of real vehicle, white noise is added to the original model outputs. The parameters of the simulation vehicle model are shown in Table I.

\section{TABLE I. MAIN PARAMETERS OF CARSIM VEHICLE MODEL}

\begin{tabular}{|l|l|l|l|}
\hline \multicolumn{1}{|c|}{ Symbol } & \multicolumn{1}{|c|}{ Quantity } & \multicolumn{1}{c|}{ Value } & \multicolumn{1}{c|}{ units } \\
\hline$I_{z}$ & $\begin{array}{l}\text { Moment of } \\
\text { vehicle inertia }\end{array}$ & 2031.4 & $\mathrm{~kg} . \mathrm{m} 2$ \\
\hline$a$ & $\begin{array}{l}\text { Chassis length of } \\
\text { front }\end{array}$ & 1.04 & $\mathrm{~m}$ \\
\hline$b$ & $\begin{array}{l}\text { Chassis length of } \\
\text { rear }\end{array}$ & 1.56 & $\mathrm{~m}$ \\
\hline$m$ & Vehicle mass & 1111 & $\mathrm{~kg}$ \\
\hline & Size of tire & $185 / 65 \mathrm{R} 15$ & \\
\hline
\end{tabular}

The vehicle speed is $120 \mathrm{~km} / \mathrm{h}$, and the adhesion coefficient $\mu$ is 0.85 in this simulation. In order to evaluate the performance of the proposed feedback observer, the simulation under different observer gains is conducted through sinusoidal input and step input test maneuver. Fig. 5 and Fig. 6 present observer results for the two tests.

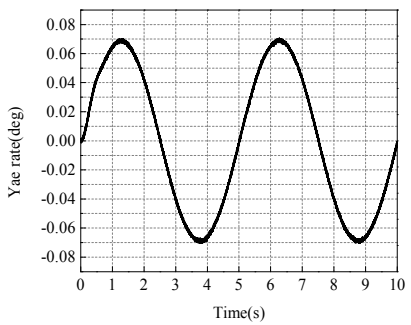

(a) yaw rate

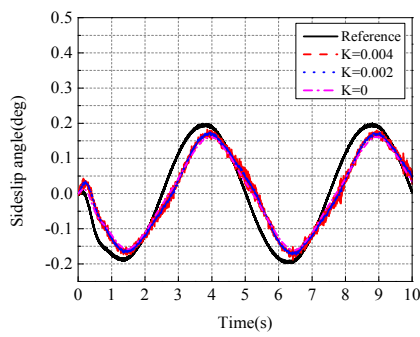

(b) sideslip angle ( $K$ is nonnegative)

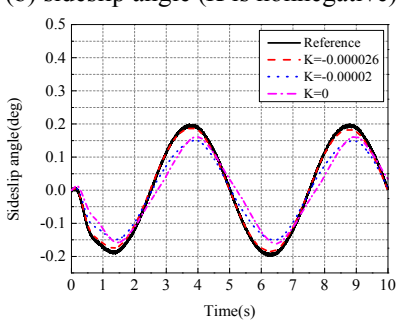

(c) sideslip angle ( $K$ is nonpositive)

FIGURE V. SIMULATION RESULTS OF SINUSOIDAL INPUT

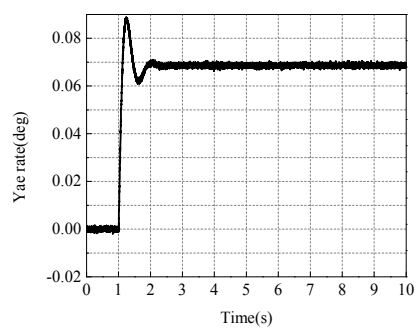

(a) yaw rate

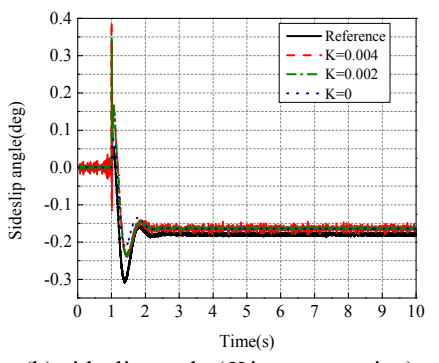

(b) sideslip angle ( $K$ is nonnegative) 


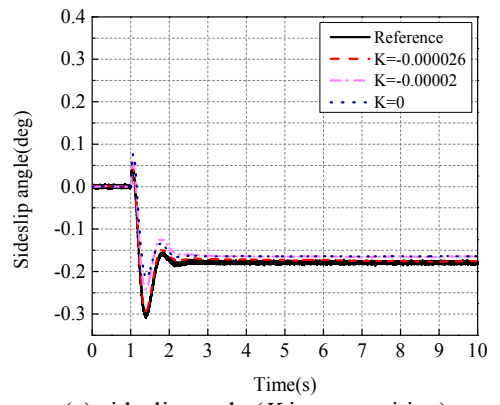

(c) sideslip angle ( $K$ is nonpositive)

FIGURE VI. SIMULATION RESULTS OF STEP INPUT

In the sinusoidal test (Fig. 5), estimation results are relatively satisfactory when observer gain $K=-2.6 \mathrm{E}-05$, nevertheless significant differences appear between sideslip angle estimations and measurement (normalised mean errors of $12 \%$ for $K=0.002$ and $10 \%$ for $K=0.004$ ). The estimated values ripple is eliminated when $K$ is non positive as shown in Fig. 5(c).

The test results of step test are shown in Fig. 6. From Fig. 6(b) and Fig. 6(c), it can be seen that when observer gain

$K=-2.6 \mathrm{E}-05$, the estimated sideslip angle matches the reference more accurately. And ripples still exists at the beginning of the step when $K=0.002$ and $K=0.004$ (Fig. 6(c)).

It can be seen that for each tests, the estimated values show good coherences with the outputs from CarSim when $K=-$ 2.6E-05. The estimated values show more and more coherences with the outputs from CarSim with the change of observer gain from $-2.0 \mathrm{E}-05$ to $-2.6 \mathrm{E}-05$. Indeed, only the appropriate observer gain will make correction for interference error and improve the estimation accuracy of the observer.

\section{CONCLUSIONS}

This paper focuses on improving the estimation accuracy of sideslip angle by designing a feedback observer. Based on a 2-DOF vehicle model, a feedback observer is built with the correction item, which adopts the three-map of tire lateral force to improve estimation accuracy of feedback observer and reduce the computational burden. Simulation results show the feedback observer has higher estimation accuracy and the calculation is simple. Therefore, the estimation method of sideslip angle proposed in this paper will find wider applications in vehicle stability control.

\section{ACKNOWLEDGMENT}

The work is supported by the Jilin Province Science and Technology Found for Young Scholars (No.20170520097JH; No.20160520106JH), and by the Key Technology and Development Program of Jilin Province (No.20170201005GX), by the Special Project for Construction Production-Study-Research Cooperation Innovation Demonstration Sites in Changchun City, by the Science and Technology Planning Project of Changchun City (No. 16CX21).

\section{REFERENCES}

[1] Li L, Ran X, Wu K, Han JSZ, “ A novel fuzzy logic correctional algorithm for traction control systems on uneven low-friction road conditions," Veh Syst Dyn, 2015, pp. 711-733

[2] Li L, Jia G, Chen J, Zhu H, Cao D, Song J , “ A novel vehicle dynamics stability control algorithm based on the hierarchical strategy with constrain of nonlinear Tyre forces," Veh Syst Dyn, 2015, pp.1093-1116

[3] AT Van Zanten, "Bosch ESP system: 5 years of experience," Proceedings of SAE Automotive Dynamics, Stability and Controls Conference and Exhibition. Troy, Michigan, USA: SAE International, 2000-01-1633.

[4] Marcus Hiemer, Anne von Vietinghoff, Uwe Kiencke, Takanori Matsunaga, "Determination of the vehicle body side slip angle with non-linear observer strategies," Proceedings of SAE 2000 World Congress. Detroit, 2005, pp.2005-01-0400.

[5] Joanny Stephant, Ali Charara, Dominique Meizel, "Evaluation of a sliding mode observer for vehicle sideslip angle, " Control Engineering Practice, 2007, pp. $803-812$.

[6] Bevly, D. M., Ryu, J. and Gerdes, J. C, "Integrating INS sensors with GPS measurements for continuous estimation of vehicle sideslip, roll, and tire cornering stiffness," IEEE Transs, Intelligent Transportation Systemsl, 2006, pp. 483-493.

[7] Huang GB, Saratchandran P, Sundararajan N, "A generalized growing and pruning RBF (GGAP-RBF) neural network for function approximation," IEEE T Neural Networ , 2005, pp.57-67

[8] Shuming SHI, Lupker H, Bremmer P, Zuurbier J, "Estimation of vehicle side slip angle based on fuzzy logic," Automotive. Engineering, 2005, pp.426-430

[9] Milanese, M., Regruto, D. and Fortina, A., "Direct virtual sensor (DVS) design in vehicle sideslip angle estimation," American Control Conference, 2007, pp. 3654-3658.

[10] Wu J, Zhao Y, Ji X, Liu Y, Zhang L, "Generalized internal model robust control for active front steering intervention," Chin J Mech Eng-En, 2015, pp.285-2933. 